\title{
Numerical simulation of rock test for engineering properties using Mesh-free method
}

\author{
Junichi TAKEKAWA ${ }^{1}$, Yasuhiro YAMADA ${ }^{1}$, Hitoshi MIKADA ${ }^{1}$ and Yuzuru ASHIDA ${ }^{1}$ \\ ${ }^{1}$ Civil and Earth Resources Eng., Kyoto University \\ E-mail : takekawa@tansa.kumst.kyoto-u.ac.jp
}

\begin{abstract}
An element free Galerkin method which does not require connectivity between elements and nodes is examined its efficiency for rock experiment. In this study, uni-axial tensile and compressive tests and collapse of cavity are examined. In this process, the rock specimen is assumed to heterogeneous assigned by Weibull distribution. Acoustic Emission which is microscopic behavior of the specimen agrees with laboratory tests, and failure mode of the specimen also agrees with general laboratory test results.
\end{abstract}

\section{INTRODUCTION}

Recently, interest to underground spaces is increasing because of depletion of underground natural resources, geological disposal of radioactive waste and increase of earthquake hazards. Herewith, it becomes essential to understand underground rock properties. One of the most important rock properties is rock failure characteristics. The macroscopic failure of a rock is dominated by microscopic fractures generated prior to a macroscopic failure. Consequently, understanding the mechanism of the microscopic fractures is essential to figure out mechanical and engineering properties of a rock.

To understand rock failure, the statistical continuum damage model comes into being applied to rock mechanics problems in order to represent the behavior of a rock comprehensively. This statistical model has been solved by the Finite Difference Method (FDM) (Fang and Harrison,2002) and the Finite Element Method (FEM) (Tang et al.,2000). Here, we attempt to apply the mesh-free method called EFGM(Element Free Galerkin Method) (Belytschko et al.,1994) to the statistical continuum model. The main advantage of EFGM is that the connectivity between nodes and elements is not required unlike FEM. There are some other advantages in EFGM, e.g. the stress field is obtained continuously by choosing the weighting function appropriately, large deformation problems can be calculated, and so on. However, EFGM is still under development toward the practical use of engineering fields. It is, therefore, significant to examine the applicability of this mesh-free method in many engineering fields. We apply this method to numerical simulations of the uni-axial tensile and compressive tests and collapse of cavity test in order to examine the applicability of EFGM to rock mechanics and rock engineering.

\section{EFGM}

Element free Galerkin method is proposed by Belytschko in 1994. Element free Galerkin method is based on the concept of moving least-square approximation. Interpolating function $\mathbf{u}^{\mathrm{h}}(\mathrm{x})$ is expressed using moving least-square approximation as follows,

$$
u^{h}(x)=p^{T}(x) a(x)
$$

where $\mathbf{p}^{\mathrm{T}}(\mathrm{x})$ is base function and $\mathbf{a}(\mathrm{x})$ is a vector of unknown parameters depending on coordinate system. A vector $\mathbf{a}(\mathrm{x})$ is determined by minimizing the discrete weighted square difference between approximated value and real value.

$$
J=\sum_{I}^{n} w_{I}(x)\left[p^{T}(x) \cdot a(x)-u_{I}\right]^{2}
$$

where $\mathbf{w}_{\mathrm{I}}(\mathrm{x})$ is weight function. Substituting $\mathrm{a}(\mathrm{x})$ determined by equation (2) into equation (1), interpolating function can be obtained, 


$$
u^{h}(x)=\sum_{I}^{n} \phi_{I}(x) u_{I}
$$

where the vector $\phi_{I}(x)$ is the shape function.

The weak form of the equilibrium is given as follows;

$$
\int_{V} \sigma \delta \varepsilon d V-\int_{S} f \delta u d S-\int_{S} \alpha(u-\bar{u}) \delta u d S=0
$$

where $\sigma$ is the stress tensor, $\varepsilon$ is the strain tensor, $\mathbf{u}$ is the displacement vector and $\alpha$ is penalty coefficient. Penalty coefficient is used in penalty method in order to satisfy the essential boundary condition.

In this study, Lagrangian kernel expressed in terms of material coordinates is used as follows,

$$
W_{I}(X)=W\left(X-X_{I}, h_{0}\right)
$$

For Lagrangian kernel, the neighborhood particles of influence do not change during the course of the simulation. Another kernel called Eulerian kernel is defined in terms of spatial coordinate. So the shape function in Eulerrian kernel must be calculated from step to step, however, that of Lagrangian kernel is calculated only at once in first step.

\section{PARAMETERS OF THE SPECIMEN}

Young's modulus is 30GPa, poisson's ratio is 0.25 , cohesion is $20 \mathrm{MPa}$, internal friction angle is 35 degrees. Failure criterion used in this study is Mohr-Coulomb criterion with tensile cutoff. Cohesion and tensile strength are distributed in order to express heterogeneity following Weibull distribution. Probability density function of Weibull distribution is expressed as follows;

$$
f(x)=\frac{m}{x_{0}}\left(\frac{x}{x_{0}}\right)^{m-1} \exp \left[-\left(\frac{x}{x_{0}}\right)^{m}\right]
$$

where $\mathrm{m}$ is the shape parameter and $x_{0}$ is scale parameter. Fig. 1 shows the probability density function of Weibull distribution changing various shape parameters. It can be seen that the probability density function is focused around unity with increase in large number of shape parameter.

In this study, failure criterion is assessed at each integration point. In addition, the failure is brittle

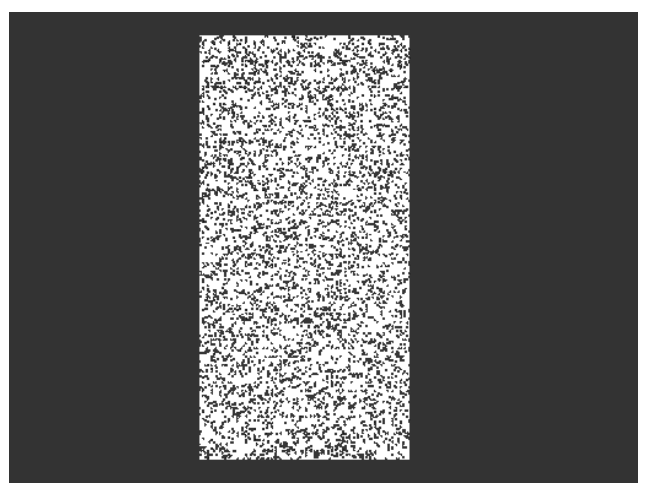

(a) Microscopic failure points are generated internal the specimen.

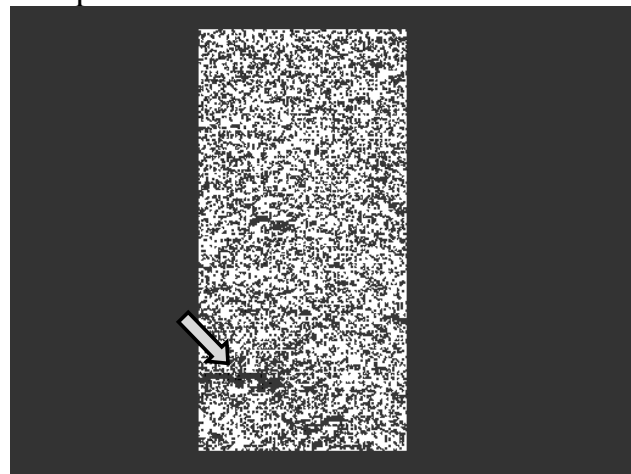

(b) The localization of microscopic failure is occurred.

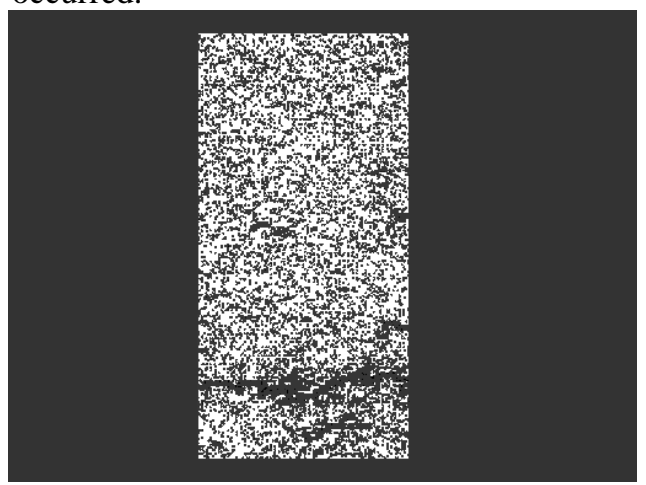

(c) Macroscopic failure plane is formed.

Fig.1 The developing process of microscopic failure.

failure with no plastic range. When brittle failure is occurred in the specimen, the stress is redistributed around neighbor integration point. Then the neighbor integration points are satisfied failure criterion, the stresses are calculated without increasing further displacement.

\section{SIMULATION RESULTS}

\section{(1) Uni-axial tensile test}

Fig. 1 shows the failure patterns of the uniaxial tensile test with the shape parameter of 1.5. The white box represents the specimen and the black points inside of the specimen represent the failure points. The analysis nodes are set in a reticular pattern and the square background cells are 


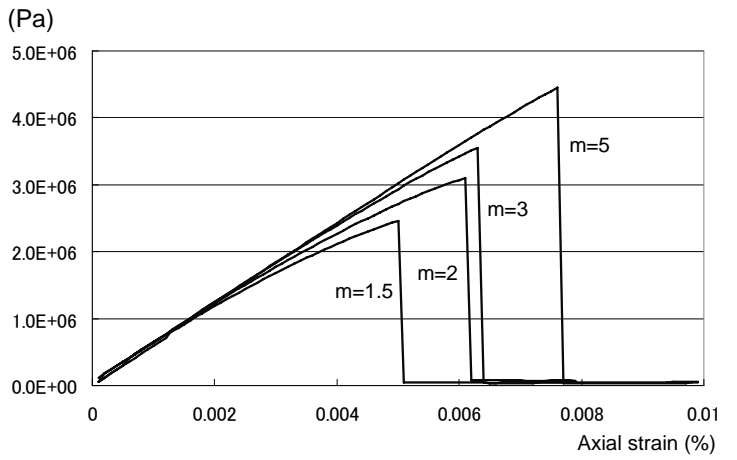

Fig.2 Stress-strain curves of uniaxial tensile test.

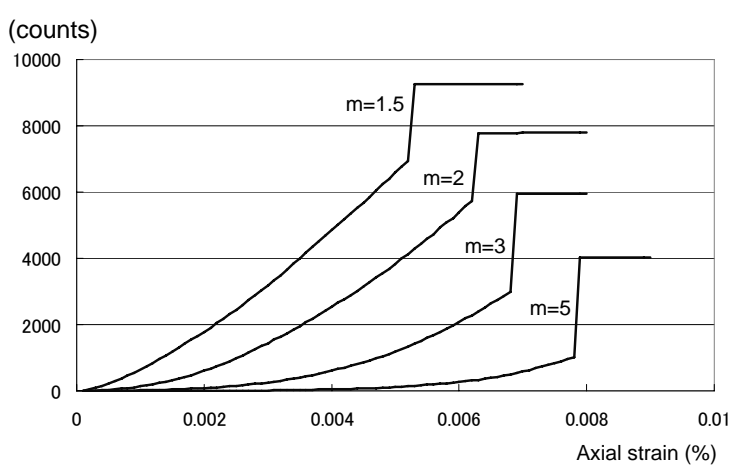

Fig.3 AE counts-strain curves of uniaxial tensile test.

collocated so that their corners coincide with the nodal locations. The size of the specimen is $5 \times 10 \mathrm{~cm}$, and the applied external displacement is $0.0001 \mathrm{~mm} / \mathrm{step}$ constantly. In Fig.1(a), the microscopic failures are generated in the whole specimen. By successive loading, the microscopic failures are localized at one point in the specimen indicated by the arrow in Fig.1(b), and this localization develops and propagates coalescing with neighboring microscopic failures to create a macroscopic failure (Fig.3(c)). This developing process and its direction perpendicular to the loading direction in this simulation correspond with those in the laboratory test.

Fig. 2 shows the stress-strain curves and Fig. 3 shows the AE counts-strain curves in the uni-axial tensile tests. It can be seen that the peak strength of the uni-axial tensile tests increases with an increase in the shape parameter. It can be an explanation for this result that a specimen with a large shape parameter is more homogeneous than that with a small shape parameter. This is supported by AE events shown in Fig.3, i.e. AE counts-strain curves for the large shape parameters show more drastic increases of AE counts. Furthermore the stress-strain curve with the larger shape parameter has steeper rise than that with the smaller shape parameter. This is considered that the stiffness of the specimen decreases with an increase in microscopic failures

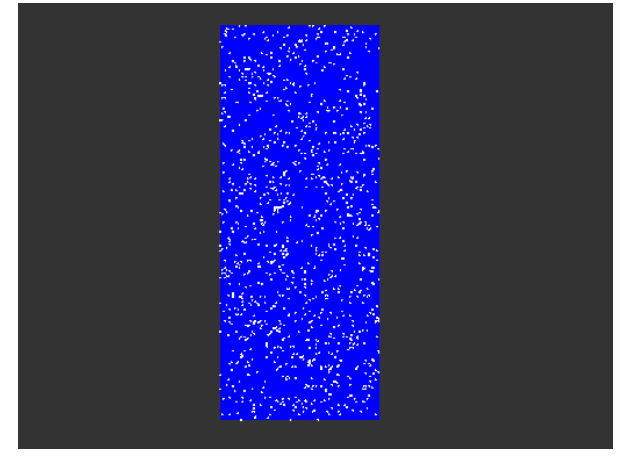

(a) Microscopic failure points are generated internal the specimen.

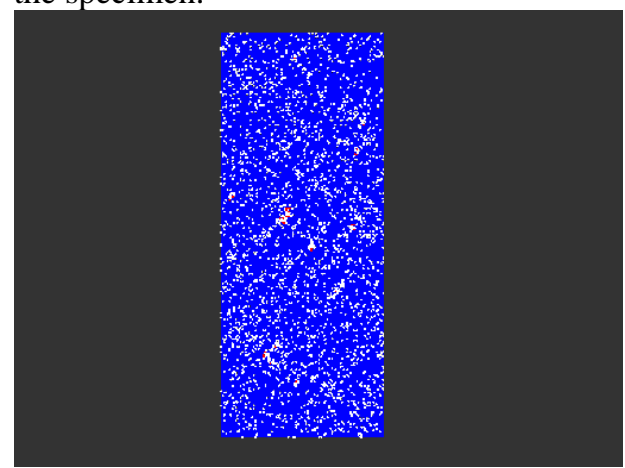

(b) The localization of microscopic failure is occurred.

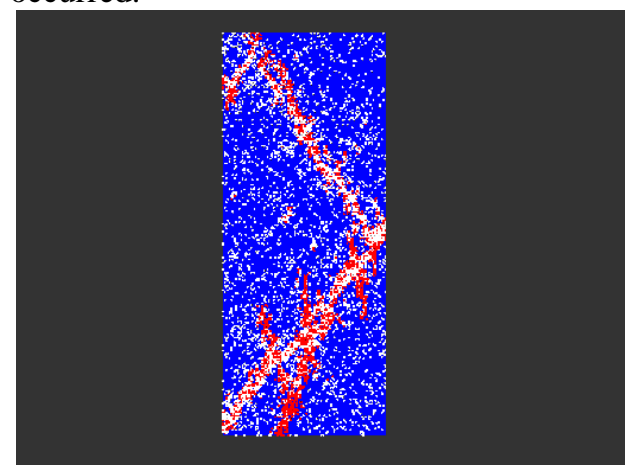

(c) Macroscopic failure plane is formed.

Fig.4 The developing process of microscopic failure.

because of crack interaction. A number of simulation results with different input values of random number show the similar pattern described above.

\section{(2) Uni-axial compressive test}

Uni-axial compressive tests are carried out in order to investigate mechanical and engineering properties of heterogeneous specimen. The size of the specimen is $4 \times 10 \mathrm{~cm}$, and applied external displacement is $0.001 \mathrm{~mm} / \mathrm{step}$ constantly. Fig. 4 shows failure mode of the specimen after uni-axial compressive test with shape parameter of 2.5. In Fig.4, white points denote failure points in shear mode, and red one denote failure points in tensile mode. In many laboratory tests, the specimen ruptures along loading direction generally. In this study, the specimen failures in tensile criterion from 


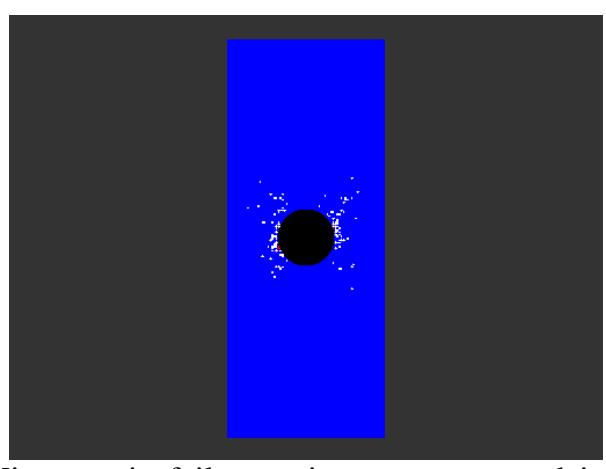

(a) Microscopic failure points are generated internal the specimen.

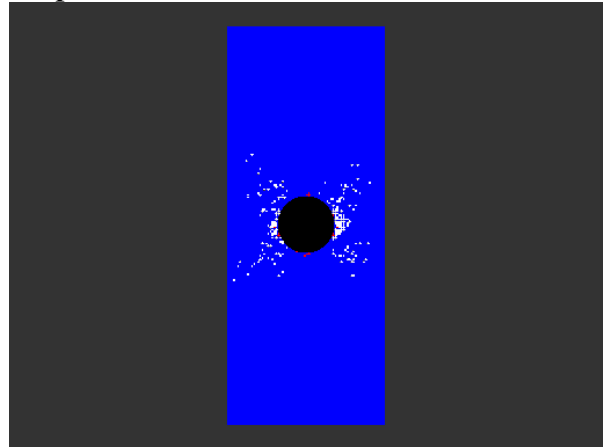

(b) The localization of microscopic failure is occurred.

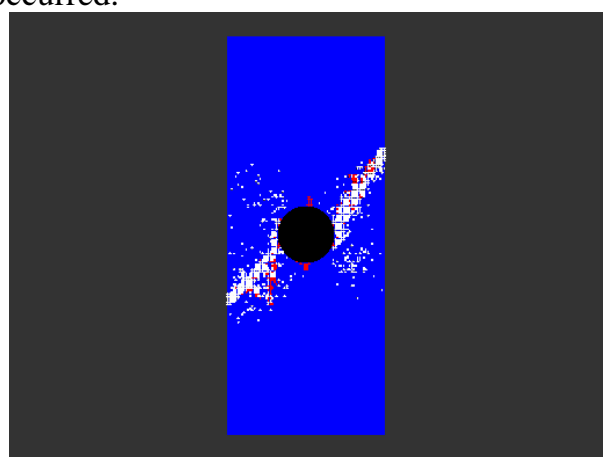

(c) Macroscopic failure plane is formed.

Fig.5 The developing process of microscopic failure.

a microscopic viewpoint. This shows the process of rock failure is represented clearly.

\section{(3) Collapse of cavity}

Next, the evolution of fracture around a cavity is examined through a numerical simulation. Confining pressure is applied at side wall of the specimen. Mechanical parameters of the specimen are similar to uni-axial tensile and compressive tests. Macroscopic failure pattern is shown in Fig.5. In Fig.5(a), microscopic failures occur at side wall of the circular hole. In fig.5(b), remote failures appear all around the circular hole, and finally, a macroscopic failure plane across the specimen is formed. This failure process is very similar to laboratory test result (Fakhimi et al.,2002).

\section{CONCLUSIONS}

In this study, we investigate the applicability of EFGM to rock mechanics by conducting numerical experiments. Our simulation results in this paper show the followings.

1. The sequential behavior of rock failure in the uni-axial tensile and compressive tests is represented by our numerical simulation method which adopts a statistical continuum damage model.

2. The evolution of failure around a cavity is similar to laboratory test result. This shows the applicability of EFGM under a general stress condition.

The greatest characteristic of EFGM is that the shape function is determined by a moving least-squares method without using an element structure. However, it is easy to incorporate this concept into existing FEM codes because EFGM is based on Galerkin method same as FEM. This suggests that a number of contributions have been made in the past by means of FEM can be also utilized in future studies by means of EFGM which is brought to the attention recently.

\section{REFERENCES}

1) Belytschko, T., Lu, Y. Y., Gu, L., 1994, Element-Free Galerkin Methods, Int. J. Num. Meth. Eng., 37, 229-256.

2) Fakhimi, A., Carvalho, F., Ishida, T., Labuz, J. F. (2002) : Simulation of failure around a circular opening in rock, Int. J. Rock Mech. Min. Sci., 39, 507-515.

3) Fang, Z., Harrison, J. P., 2002, Development of a local degradation approach to the modeling of brittle fracture in heterogeneous rocks, Int. J. Rock Mech. Min. Sci., 39, 443-457.

4) Tang, C. A., Liu, H., Lee, P. K. K., Tsui, Y., Tham, L. G., 2000, Numerical studies of the influence of microstructure on rock failure in uniaxial compression - Part1:effect of heterogeneity, Int. J. Rock Mech. Min. Sci., 37, 555-569. 